\title{
Parietal Lobe
}

National Cancer Institute

\section{Source}

National Cancer Institute. Parietal Lobe. NCI Thesaurus. Code C12354.

One of the lobes of the cerebral hemisphere located superiorly to the occipital lobe and posteriorly to the frontal lobe. Cognition and visuospatial processing are its main functions. 